\title{
Age of Acquisition Effects in Chinese EFL learners' Delexicalized Verb and Collocation Acquisition
}

\author{
Miao Haiyan \\ School of Foreign Languages, Jiangxi Normal University \\ No. 99, Ziyang Road, Nanchang, China \\ E-mail: salomehy@aliyun.com
}

\author{
Received: 14-10- 2014 \\ Accepted: 07-01- 2015 \\ Advance Access Published: January 2015 \\ Published: 01-05- 2015 \\ doi:10.7575/aiac.ijalel.v.4n.3p.189 \\ URL: http://dx.doi.org/10.7575/aiac.ijalel.v.4n.3p.189
}

The research is financed by the Reform Project in Teaching of Jiangxi Normal University.

\begin{abstract}
This paper investigates age of acquisition (AoA) effects and the acquisition of delexicalized verbs and collocations in Chinese EFL learners, and explores the underlying reasons from the connectionist model for these learners' acquisition characteristics. The data were collected through a translation test consisted of delexialized verb information section and English-Chinese and Chinese-English collocation parts, aiming to focus on Chinese EFL learners' receptive and productive abilities respectively. As Chinese EFL is a nationally classroom-based practice beginning from early primary school, the pedagogical value and different phases of acquisition are thus taken into consideration in designing the translation test. Research results show that the effects of AoA are significant not only in the learners' acquisition of individual delexicalized verbs but also in delexicalized collocations. Although learners have long begun to learn delexicalized verbs, their production indicates that early learning does not guarantee total acquisition, because their grasp of delexicalized verbs still stay at the senior middle school level. AoA effects significantly affect the recognition but not the production of collocations. Furthermore, a plateau effect occurs in learners' acquisition of college-level delexicalized collocations, as their recognition and production have no processing advantages over earlier learned collocations.
\end{abstract}

Keywords: Chinese EFL; AoA effects; delexicalized; collocations

\section{Introduction}

Age is an important concept in second language acquisition (L2A) and foreign language learning. For years, researchers have been trying to find out differences between second language acquisition (L2A), foreign language learning and native language acquisition from the perspective of age. It is usually suggested in numerous studies that age play a decisive role in distinguishing achievements by L2A, foreign language learning and native language acquisition. Generally speaking, research on age in language learning and acquisition concentrates more on morphosyntax and phonology. It is only in recent decades that the relationship between lexical acquisition and age begins to attract attention, and as a result age of lexical acquisition and its effects have become one of the most concerned questions by psycholinguists. For word learning, age of acquisition (AoA) refers to the age at which an individual makes contact with the oral or written word form and understand its meaning. Research shows that AoA is closely related to lexical acquisition (Zevin \& Seidenberg, 2002).

This study investigates age of acquisition (AoA) effects and the acquisition of delexicalized verbs and collocations in Chinese EFL learners, and tries to explore the underlying reasons from the connectionist model for these learners' acquisition characteristics. This study employs a translation test to probe into in-depth analysis of Chinese EFL learners' usage and acquisition of delexicalized verbs and collocations. To achieve this goal, we draw on the connectionist model and the concept of AoA. Relevant literature and key notions underlying the study will be introduced first, and research design will be explained before the research findings are presented and discussion in detail.

\section{Research background}

The connectionist model (see Christiansen \& Charter, 2001) proposes that learner grammar is a complex network made up by many neuron-like processing units or nodes. In this network, each node is linked to other many nodes, but the linking strength varies. This strength can be either strengthened or weakened through language exposure. The connectionist model has been widely applied to many areas of study, including areas of language learning and specifically L2A. In the process of language learning, language items are individual nodes stored in the learners' mind. These individual nodes can be either isolated or connected units. The isolated nodes are those that bear no relevance to other nodes. On the contrary, the connected nodes are those that are related to other nodes. It is through repeated language exposure and contact that relevance is established between the nodes. In fact, the more the number of links established, the more likely the connection strength between nodes will be enhanced. Then language learning will occur 
and language acquisition will become preempted, thus excluding the possibility of other interfering language factors. On the contrary, if non-relevant node weight is added into this process, learning will become more difficult, slow down, or even never happen, because learners have to spend more time to notice and learn this specific language item. In this case, language learning does not necessarily lead to language acquisition.

Set within the connectionist model, AoA research attempts to explain the relationship between age and acquisition, and offer insights into how to make best use of age in the optimization of acquisition. Recent decades have witnessed a growing number of studies on AoA and its effects, and there are several AoA hypotheses put forward by different scholars. One of the most recent and influential theory is the arbitrary mapping hypothesis in the field of AoA. According to this hypothesis, AoA effects refer to the phenomenon that early acquired words have processing advantages over later ones, which means that earlier acquired words are more easily recognized and produced. Since AoA effects affect word recognition and production, the connectionist model explains AoA effects through such concepts as lexical network and lexical representations. Based on the connectionist model, the AoA effects can well reflect the mapping relationship between formation of the lexical network, and the input (e.g. morphology) and output (e.g. phonology and semantics) representations. It is found out that there is great homogeneity in the mapping between early acquired words. Thus it is relatively much effortless to recognize and produce these early acquired words. If later acquired words can be made relevant to these earlier ones, then there will be small or even no AoA effects, because the relevance can facilitate the recognition or the production of later acquired words. Otherwise, AoA effects will increase, making recognition and production of later acquired words difficult or impossible. This arbitrary mapping hypothesis has been tested and verified by empirical studies. For example, Zevin \& Seidenberg (2002) revisits and modifies this hypothesis by providing ample computation modeling evidence.

Research into AoA and its effects has been attracting much attention from both psychology and L2A. It should be pointed out that AoA and its effects first attracted psychologists' interest. Gilhooly \& Logie (1980) collected subjective AoA ratings for nearly 2000 words----familiarity, imageability and concreteness. These ratings have also been adopted by other researchers in testing AoA effects in tasks like lexical decision and object naming (Coltheart, Laxon \& Keating, 1988; Ellis \&Morrison, 1998). Zevin \& Seidenberg (2002), based on computer modeling evidence, explored AoA effects in word reading. They found that AoA effects would occur if later acquired words could not establish relevance with earlier ones. Liu, et al. (2008) investigated AoA effects in spoken Chinese. Their study reveals that it is in written rather than spoken Chinese that AoA effects are detected, which well tests the generalizability of AoA effects in languages other than alphabetic languages like English. Because AoA effects are proved to be an important issue, AoA effects begin to arouse interest from the sphere of L2A. As a result, the phenomenon of AoA effects is believed to occupy a significant role in second language acquisition. Numerous studies have been carried out on AoA and its effects in L2A (for review, see Birdsong, 2006). It is widely recognized that AoA is predictive of L2A outcomes (Birdsong, 2006). In fact, comparison between early and late arrivers in the target language country shows that the earlier a word is acquired, the more processing advantage there will be, thus suggesting better L2A outcome therein (Birdsong, 2006). However, it is worth mentioning that these above studies mainly use psychological mapping methods or multivariate regression analysis. Subjects are either English native speakers or second language learners from English-speaking countries. More research is needed to probe into learners with other language backgrounds, of other types or of different language proficiencies, so that the applicability of AoA effects can be further verified and related hypotheses can be modified accordingly.

In China, scholars are also beginning to recognize the significance of AoA and its effects. Wang Chuming (2001) reviewed major findings and the latest development of research on age in L2A both home and abroad. He further suggested future research possibilities in L2A and pedagogical implications for EFL in China. Chen Baoguo, You Wenping and Wang Lixin (2004) introduced AoA and AoA effects, and further tracked the progresses achieved in this sphere of research in psychology in western countries. Zhao Fei and Zou Weicheng (2008), in an attempt to relate AoA effects with foreign language learning, conducted a study on the relationship between foreign language learning and the age of onset. There are also studies trying to employ psychological experimental task to relate AoA effects with lexical representations. For instance, Lu Zhi and Tu liu (2010) carried out a bilingual stroop task to illustrate the relation between AoA in L2 and proficient Chinese-English bilinguals' mental lexicon representation. Their research verifies the limited effects of age on L2A from the perspective of cognitive science. Studies concerning AoA and its effects have developed rapidly in China, but they mainly concentrate on introducing concepts, literature review, or general relationship between AoA and EFL (or L2A). It needs to be pointed out that these studies seem to have neglected the specific phases of language learning. Consequently, AoA effects are treated as an end state instead of a process. The reason lying behind is that AoA and its effects are generally analyzed independently of what learners have actually gone through. Although it is widely recognized now by Chinese scholars that AoA and lexical acquisition are closely linked, research methods and how to integrate pedagogical value into AoA still remain hotly debated.

Therefore, as an explorative study, the present paper aims to explore AoA effects in Chinese English learners' lexical acquisition, focusing on AoA effects in both word and collocation acquisitions. In our paper, the pedagogical value and specific acquisition phase will be given full play to. The pedagogical value and specific acquisition phase are closely interwoven, since the pedagogical value differs in different specific acquisition phases. By comparing pedagogical values in specific acquisition phase, we would be in a better position to spot the connection between AoA effects and lexical acquisition. It is hoped that our study can reveal implications for classroom-based EFL learning in countries such as China, thus further prove the generalizability of AoA effects in lexical acquisition. 


\section{The study}

\subsection{Subject}

The study draws on data from research work conducted in a normal university at a provincial level in mainland China. This university, which has a history of more than one hundred years, is a government-subsidied university with a national enrollment. Only after passing the National College entrance examination, students are entitled four years of study for bachelor's degrees in the university. For their first two years in college, students have to take the compulsory course College English, which counts two credits each semester. At the end of the second year, students are supposed to be able to pass the National College English Test (CET 4). Generally speaking, CET4 is taken as the norm adopted by many colleges and universities for whether students have achieved successfully college English proficiency in China. CET 4 exam takes place two times every year, usually at the end of each semester in June or December.

The subjects are 161 students from three natural classes in this university. Survey of the students' personal information indicates that these students show great homogeneity in their experience of classroom-based English learning since primary school, which proves the powerfulness and the uniformity of national English as the foreign language education program in China. The students' mother tongue is all Chinese. At the time of this research, these students are nearing the end of the second college year. All of these college students have already taken the examination of CET 4. This provincial college stipulates that students take English courses at the end of the first year in college, which is also a policy in the majority of colleges in China. If there are students who fail to pass CET 4 in the first year, they are allowed to retake the exam every semester. All this means that the subjects have finished their classroom English learning in college, or most probably their learning of English forever if they do not go on for post-graduate studies, because post-graduates are required EFL learning for one year.

\subsection{Target words}

The choice of target words abide by the criterions of the pedagogical value and the time phase of AoA. By consulting English teaching syllabuses from middle school to college, we finally choose five typical delexicalized verbs do, give, have, make, take and their collocations (Collins CoBuild English Grammar, 1990) as the target words. These five words are required by both the middle school English teaching syllabus and college English syllabus. For the collocations, the junior middle school syllabus doesn't include the requirement of delexicalized verb collocations, so we just listed the delexicalized verb collocations required by the two syllabuses of senior middle school and college. There are altogether 69 such collocations for the senior middle school level and 55 for the college level, wherein 16 collocations from middle school syllabus overlap with those from college. Thus if the reoccurring collocations are excluded, the subjects are supposed to acquire 108 different delexicalized verb collocations up to the college level.

The reason why we choose delexicalized verbs and their collocations is that delexicalized verbs are high-frequency verbs widely used in English. However, even though these verbs are highly frequent and also very important, they themselves do not have clear lexical meanings. Instead, the meanings of delexicalized verbs are closely related with their collocates and contexts. Lewis (1997) pointed out that the acquisition of delexicalized verb collocations is an important aspect of vocabulary teaching, because its acquisition follows the principle of chunk usage----being used, stored and retrieved as whole.

\subsection{Instrument}

For the research instrument, we designed a translation test based on Webb (2008). In order to completely survey the subjects' acquisition, this translation test also includes sections of personal information and delexicalized verb information besides delexicalized verb collocations. For personal information, subjects were supposed to give information like age, sex, onset of age of English learning and their English coursebooks used at different English learning stages. In the part of delexicalized verb information, subjects were asked to provide parts of speech, different forms (past tense form, past participle form) and meanings. Included in the section of delexicalized verb collocations are the receptive translation test and the productive translation test. The receptive translation test requires subjects to offer the Chinese meanings of English delexicalized verb collocations, with the aim to test the subjects' comprehension. The productive translation test, on the other hand, demands subjects to provide the English delexicalized verb collocations in accordance with their Chinese meaning equivalents, so that the subjects' productive ability can be tested.

Through random sampling, we assigned the 108 delexicalized verb collocations to the receptive translation test and the productive translation test respectively. The receptive translation test includes 34 delexicalized verb collocations learned in middle school, and 20 delexicalized verb collocations at the college level. The productive translation test contains 35 delexicalized verb collocations learned in middle school, and 19 delexicalized verb collocations at the college level. For the rating of the collocation translation test, each collocation counts 1 point. Correct answer gets 1 point, and wrong or no answer is 0 point. If there is any spelling mistake in a discernible correct answer, then the answer gets 0.5 point. Thus the receptive and productive translation test each has 54 point, totaling 108 point for the whole collocation translation test.

\subsection{Procedure}

Before the test, the author explained the purpose and the steps of taking the test. The test did not begin until the subjects fully understand the procedures involved. The subjects were asked to write answers in the blank of the test paper, in the process of which consultation of dictionaries or reference books is forbidden. The test lasted for 35 minutes. Altogether we collected 161 copies of test paper, with 49 copies invalid copies. The invalid copies are either incomplete or belong 
to subjects who didn't pass CET 4. Because our study needs to distinguish clearly between the different acquisition stages (middle school and college English learning), translation test papers from students who failed in CET 4 are thus excluded. Therefore, we have altogether 112 copies of valid translation test papers.

\subsection{Statistical analysis}

After the collection of the test papers, all the items in the test were carefully counted and analyzed. The acquisition of delexicalized verb information was carefully counted and then calculated in percentages. Paired T-test was employed to see if there was any difference in delexicalized verb collocations between the receptive test and the productive test. Then delexicalized verb collocations were divided into 3 types on the basis of different acquisition stages: middle school/college overlapping ones (overlapping highlights the pedagogical value and significance, and that's the reason why this overlapping type is distinguished), senior middle school level, college level. Further analysis was carried out by means of ANOVA, in order to find out AoA effects in comprehension and production of delexicalized verb collocations at different learning phases.

\section{Results}

\subsection{Delexicalized verbs and AOA effects}

Statistical results of personal information in the test paper shows that all these subjects started to learn English from primary school, and their English coursebook was the authoritative PEP English textbook, which is adopted as English coursebooks in primary school nation-wide. This means that the requirements for the subjects' English learning are basically the same.

Based on Full-time Senior Middle School English Teaching Syllabus and College English Curriculum Requirements and Syllabus, we summarized and counted delexicalized verb information needed to acquire in both senior middle school and college. For the delexicalized verb information, different forms should be acquired in the senior middle school syllabus. However, the delexicalized verb form information is not even mentioned in the college requirements and syllabus, which indicates that students should grasp different forms of delexicalized verbs very well before they enter college. It is also worth pointing out that the parts of speech and meanings of delexicalized verbs undergo different changes and demands in college English. Table 1 provides the detailed delexicalized verb information of form, parts of speech and meaning in both senior middle school and college respectively, which will enable the perception of the changes between these different acquisition phases. From Table 1, it can be seen that parts of speech begin to become more specific and precise in English acquisition at the college level, instead of being quite general in senior middle school English. Do is one of the examples, because it is treated as verb in senior middle school, but is marked as Aux. verb in college. On the other hand, there are fewer demands on the meaning aspect in college English. This proves Gui's argument (2007) that the acquisition of meaning tends to generalize for delexicalized verbs, which is consistent with the characteristics and trend of language delexicalization.

Table 1. Basic information of DV in the senior middle school and college syllabuses

\begin{tabular}{|c|c|c|}
\hline phasesDV learning & Senior middle school & College \\
\hline do & $\begin{array}{l}\text { v. (did, done) perform; finish a particular } \\
\text { activity or job }\end{array}$ & Aux.v. perform \\
\hline give & v. (gave, given) pass; provide; pay & vt. do; send \\
\hline have & $\begin{array}{l}\text { v. (had, had) include; eat; drink; carry on; } \\
\text { experience; make }\end{array}$ & $\begin{array}{l}\text { aux. v. used with the past participle of } \\
\text { another verb to make the perfect tense } \\
\text { vt. include; eat }\end{array}$ \\
\hline make & v. (made, made) do; produce & v. do; cause; manufacture \\
\hline take & $\begin{array}{l}\text { v. (took, taken) get; move sb./sth. from one place } \\
\text { to another; do (used with nouns); spend time; } \\
\text { eat; drink; have medicine; ride by car (ship) }\end{array}$ & $\begin{array}{l}\text { vt. get; grab; move sb./sth. from one } \\
\text { place to another; eat }\end{array}$ \\
\hline
\end{tabular}

(*DV stands for delexicalized verbs. The meanings for these DV are originally in Chinese, but later translated into English for the sake of convenience)

Table 2. Percentage of the acquired DV information

\begin{tabular}{lllllll}
\hline & PA & \multicolumn{2}{l}{ Parts of speech } & Verb forms & Verb meanings & \\
\cline { 2 - 6 } & v. & $\begin{array}{l}\text { Aux } \\
\text { v./vt. }\end{array}$ & Past tense & $\begin{array}{l}\text { Past } \\
\text { participle }\end{array}$ & Meaning acquired & others \\
give & $92 \%$ & $8 \%$ & $100 \%$ & $100 \%$ & $100 \%$ (perform, finish) & $0 \%$ \\
have & $100 \%$ & $0 \%$ & $100 \%$ & $100 \%$ & $98 \%$ (provide, pass) & $2 \%$ \\
make & $90 \%$ & $10 \%$ & $100 \%$ & $95 \%$ & $99 \%$ (include, eat) & $1 \%$ \\
take & $100 \%$ & $0 \%$ & $100 \%$ & $100 \%$ & $97 \%$ (do) & $3 \%$ \\
& $100 \%$ & $0 \%$ & $100 \%$ & $97 \%$ & $95 \%$ (get, move & $5 \%$ \\
\end{tabular}

(*DV stands for delexicalized verbs and PA for percentage of accuracy.) 
It can be seen from Table 1 that the acquisition of delexicalized verbs is quite imbalanced. As for parts of speech, most subjects have grasped what is required in the senior middle school syllabus, but there are only a few subjects who can provide the feature of $A u x . v$. for delexicalized verbs do and have mentioned in the college English syllabus. For verb forms, the past tense form is one hundred percent acquired by the subjects. The past participle form is also acquired pretty well, but with just very few subjects not acquiring the past participle of have and take. Although the subjects have acquired many of the meanings stipulated in the senior middle school syllabus, what they have in fact acquired is mainly restricted to one or two of the core meanings, which contradicts with the trend of generalization of delexicalized verb meanings reflected in the college syllabus. It is clear then that early acquired delexicalized verbs are better grasped than later acquired one. These early verbs have processing advantages: subjects can produce early acquired words, let alone to comprehend them. The subjects have passed the CET 4, but their grasp of delexicalized verbs still stays at the level of senior middle school. All this manifests AoA effects in the acquisition of delexicalized verbs.

\subsection{Delexicalized verb collocations and AOA effects}

Delexicalized verb collocations occupy an important place in both the senior middle school syllabus and the college syllabus. As a major focus in our study, delexicalized verb collocations are tested on their comprehension and production as reflected in the subsections of the translation test. Then when it comes to the translation test, how do the subjects acquire such high frequent collocations as delexicalized verb collocations? Is there any difference between comprehension and production?

\subsubsection{Comprehension and production of collocations}

Table 3 shows that the overall acquisition of delexicalized verb collocations is not good at all. The translation test has a total score of 108, but the average score by the subjects is only 46.1027 , with the highest score of 69 and the lowest score of 21. Taking the specific test items into consideration, we find that many subjects simply translate delexicalized verbs in the collocations literally according to the core meanings of delexicalized verbs acquired in senior middle school. These delexicalized verb collocations are not treated as chunks in the process of translation test, which directly results in low test score. There are numerous examples from the translation test. For instance, make one's way and take medicine are translated as the way of walking and fetch the medicine respectively in Chinese, while 买东西 (do some shopping) and 记在心里 (have in mind) are translated as take something and have into heart respectively in English.

The test score for both the receptive and productive subsections of the translation test is 54 . The average test score for the receptive test is 32.1161, much higher than that of the productive test 13.977. Paired T test results in Table 4 show that the two average test scores are significantly different $(\mathrm{T}=37.816, \mathrm{P}=.000)$, indicating better receptive grasp of delexicalized verb collocations than that of the productive. This suggests that comprehension exceeds production for delexicalized verb collocations, which is different from the above acquisition of delexicalized verbs characteristic of good production skills.

Table 3. Comparison of average receptive-productive translation test scores

\begin{tabular}{lllllll}
\hline & Mean & Number & Std. Dev & Std. Error & Maximum & Minimum \\
\hline Total test score & 46.1027 & 112 & 9.56065 & .90340 & 69 & 21 \\
Receptive & 32.1161 & 112 & 5.72924 & .54136 & 46 & 13 \\
Productive & 13.9777 & 112 & 5.06284 & .47839 & 24 & 3 \\
\hline
\end{tabular}

Table 4. Paired T test of average receptive-productive translation test scores

95\% Confidence Interval

\begin{tabular}{lllllllll}
\multicolumn{1}{c}{ Mean } & \multicolumn{2}{c}{ Std. Dev. Std.Error } & $\begin{array}{l}\text { Lower } \\
\text { Bound }\end{array}$ & \multicolumn{2}{l}{ Upper Bound } \\
\hline $\begin{array}{l}\text { Receptive- } \\
\text { productive }\end{array}$ & 18.13839 & 5.07609 & .47965 & 17.18794 & 19.08884 & 37.816 & 111 & .000 \\
\hline
\end{tabular}

\subsubsection{Comprehension, production of collocations and AOA effects}

Then we analyze the subjects' acquisition of the three types of delexicalized verb collocations: senior middle schoolcollege overlapping, senior middle school and college. Fig. 1 presents the average receptive and productive test scores of these three types of delexicalized verb collocations. From high to low the test scores are overlapping receptive (86.50), senior middle school receptive (84.81), senior middle school productive (35.48), college receptive (35.25), overlapping productive (33.44), and college productive (18.63). It can be seen that for various learning phases, receptive collocation test scores are always higher than those of productive tests. To be more specific, senior middle school receptive collocations are much better acquired than that of productive ( 84.81 vs 35.48$)$; college receptive collocations are also better than that of productive (35.25 vs 18.63). Another point is that both the receptive and productive delexicalized verb collocations acquired in senior middle school have higher test scores than those of at the college level. 


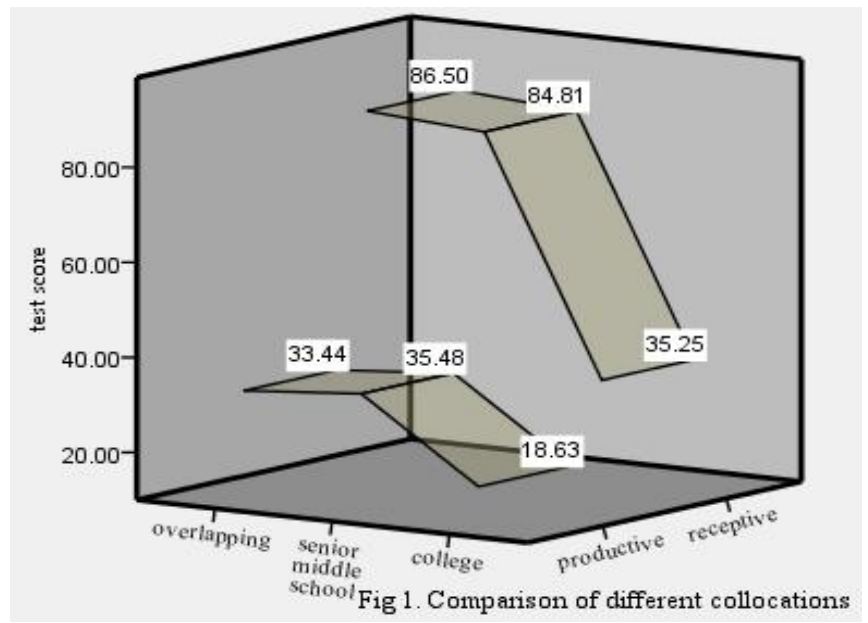

In-group and between-group ANOVA results ( presented in Table 5) show that there is significant difference between these three types of delexicalized verb collocations $(\mathrm{F}=19.485, \mathrm{p}=.000)$. In order to further detect where this difference originates, we used post hoc Scheffe tests on the average test scores of the three types of delexicalized verb collocations in both the receptive and productive translation tests. Scheffe test results in Table 6 reveal that the overlapping receptive average score (86.50) and senior middle school receptive average score (84.81) are significantly different from test scores of other types of delexicalized verb collocations, but they themselves are not significantly different from each other. For the other types of delexicalized verb collocations, there is no significant difference.

Table 5. ANOVA of receptive and productive DV collocations at different acquisition phases

\begin{tabular}{llllll}
\hline & SS & df & MS & F & Sig. \\
\hline Within-group & 72649.523 & 5 & 14529.905 & 19.485 & .000 \\
Between-group & 76060.669 & 102 & 745.693 & & \\
Total & 148710.192 & 107 & & & \\
\hline
\end{tabular}

Table 6. Post hoc Scheffe tests of receptive and productive delexicalized verb collocations

\begin{tabular}{llll}
\hline Types & \multicolumn{3}{l}{ Different acquisition phases } \\
\cline { 2 - 4 } & Overlapping & Senior middle school & college \\
Receptive & $53.06250^{*}$ & -1.69231 & $-51.25000^{*}$ \\
& 1.69231 & $51.37019^{*}$ & 1.81250 \\
& $51.01852^{*}$ & $49.32621^{*}$ & $-49.55769^{*}$ \\
& $51.25000^{*}$ & $49.55769^{*}$ & -.23148 \\
Productive & $67.868427^{*}$ & $66.17611^{*}$ & 16.61842 \\
& $-53.06250^{*}$ & $-51.01852^{*}$ & $-67.86842^{*}$ \\
& $-51.37019^{*}$ & 2.04398 & -14.80592 \\
& -2.04398 & $-49.32621^{*}$ & $-66.17611^{*}$ \\
& -1.81250 & .23148 & -16.84990 \\
& 14.80592 & 16.84990 & -16.61842 \\
\hline
\end{tabular}

Note: $\mathrm{P}<0.05$

From the above results, we can know that there is difference between comprehension and production of delexicalized verb collocations. Furthermore, the receptive and productive test scores strongly show that subjects comprehend significantly better those delexicalized verb collocations acquired in senior middle school, but they are not able to produce these delexicalized verb collocations equally well, not to mention the delexicalized verb collocations at college level. It can be drawn that AoA effects are obvious under this circumstance, because early acquired collocations seem to have more processing advantages in comprehension. When it comes to later college-acquired delexicalized verb collocations, neither comprehension nor production is satisfying. The underlying reason may be the lack of processing advantages like in those early acquired delexicalized verbs.

\section{Discussion}

Based on the above analyses of delexicalized verbs and their collocations, we have found out some characteristics of AoA in Chinese EFL learners' lexical acquisition. First and foremost, AoA exists not only in delexicalized verbs but also in their collocations. Furthermore, there is a significant difference between the comprehension and the production 
of delexicalized verb collocations. Earlier acquired delexicalized verb collocations have much more processing advantages in comprehension, suggesting strong AoA effects wherein. However, the production of delexicalized verb collocation is unsatisfactory from the very beginning, thus showing no AoA effects. Finally, later acquired delexicalized verb collocations in college English do not manifest any processing advantages.

AoA effects are evident in Chinese EFL learners' acquisition of delexicalized verbs and their collocations. Chinese EFL learners start to learn delexicalized verbs and collocations since early primary school years. With years going by, information about these verbs and collocations becomes much more varied. This information includes meanings and parts of speech of delexicalized verbs, and also relevant delexicalized verb collocations. The varied information indicates the necessity of incessant learning, in other words, students have to move from one stage to another making adaptations or modifications to their existing mental lexicon. However, early age of onset means delexicalized verbs are more likely to be generalized (Gui, 2007). This can well explain our finding that the subjects have acquired parts of speech and word forms well, but only the core meanings of delexicalized verbs. Thus, for the early acquired delexicalized verbs in the mental lexicon, connection is established between nodes of forms, meanings, parts of speech and collocations in the learners' lexical network. Early age of onset is often accompanied by simplified books or learning materials, which would usually use many delexicalized verbs and collocations for early L2A and foreign language learning (Gui, 2007). It then follows that the connection between the above early established nodes is strengthened through repeated exposure, and acquisition is usually successful. However, students still encounter these delexicalized verbs and collocations at the college level coursebooks. The later acquired delexicalized verbs in college display the following three changes. First, parts of speech become much more varied. Then meanings get more generalized. To make it even worse, the number of collocations increases enormously. Due to these changes, new nodes are created in the learners' lexical network. At this phase, the strength between these new nodes becomes decisive for the acquisition of the lexical changes. For many Chinese EFL learners, their major focus of college English classes is to pass the national CET 4 or CET 6, which serves as a proof of their English level after graduation. Therefore, it is common to see that many Chinese EFL learners are quite exam-oriented rather than usage-based. It is not strange then that the amount and frequency of language contact are far from enough, and students' existing lexical knowledge base thus often than not simply fails to stimulate the creation of nodes or nodes of low weight. The strength between these new college-level nodes is much weaker than that between the earlier connected nodes. This is why Chinese EFL learners show less processing advantages for later acquired delexicalized verbs and collocations in college than those earlier acquired ones in senior middle schools. AoA effects are obvious. The phenomenon of "First in, last out" is true for the classroom-based EFL learning in China.

Our second finding is that there is great difference between comprehension and production in the acquisition of delexicalized verb collocations, but AoA effects only significantly impact comprehension. This finding supports earlier research on features of lexical knowledge in L2A and EFL that comprehension and production is asymmetric, because comprehension and production involve different psychological and cognitive processes (Gui, 2007; Webb, 2008). Since their early English learning, Chinese EFL learners have been encountering delexicalized verb collocations very frequently in their textbooks, after-class readings or reading materials, but this encountering mainly occurs by means of reading (Gui, 2007). It is then commonplace that learners are very familiar with these delexicalized verb collocations, and thus comprehension is not difficult to them. However, what is unique of delexicalized verbs is that their meanings are vague and general, and people are much more concerned with their usages in specific contexts like collocations and formulaic expressions. Generally speaking, these collocations and formulaic expressions are highly frequent chunks, which demands separate memorization, storage and retrieval. Due to this above feature of delexicalized verb collocations, it may be difficult to establish relevance between earlier and later acquired delexicalized verb collocations, leading to great obstacles in creating connected nodes between earlier and later acquired collocations in learners' lexical network. Consequently, earlier acquired and later acquired items are stored, memorized by EFL learners as separate units. Processing advantages do exist in comprehension for earlier acquired collocations, but these processing advantages cannot be transferred to those later acquired ones. Therefore, learners perform more poorly in the comprehension task of later than earlier acquired delexicalized verb collocations, and AoA effects manifest themselves clearly. What's more, because there are limited opportunities for language contact and language use out of classroom English learning for Chinese EFL learners (Xiao \& Dai, 2004), it is less likely for the connected nodes between different acquired items get stimulated and strengthened in learners' lexical network. The result is the weak link between connected nodes, which may lead to great difficulty in production at all ages of acquisition. Thus AoA effects are not working under such circumstances.

Our finding also indicates that later acquired delexicalized verb collocations have no processing advantages in either comprehension or production. This research finding deserves our rethinking and reflection upon EFL learning and teaching at Chinese colleges. Chinese EFL learners at the college level spend a large amount of time on English learning, but why is it still difficult to produce or even comprehend these common, frequently used and early learned delexicalized verb collocations? Delexicalized verbs are lexical teddy bears, because they usually develop from their original polysemic high frequency words, through more grammaticalized, general and abstract changes, to the generation of specific meanings and collocations (Altenberg \& Cairns, 1983). These collocations are high frequency chunks abiding by Sinclair's (1991) idiom principle, namely being stored, retrieved and used as a whole. Chinese EFL learners are most probably accustomed to the learning mode of "English words plus Chinese meaning equivalents", which, combined with low-frequent language contact and stimulus in their language learning experience, leads to the practice of separating newly learned delexicalized verb collocations into isolated parts for memory and usage. Due to 
the failures to store these collocations as whole units and to establish connection with earlier acquired delexicalized verbs and collocations, nodes are not mutually accessible in learners' lexical network, and thereby comprehension obstacles and unidiomatic production are unavoidable. Just as Myles (1999) has pointed out, it is in the process of learners' disentangling contradictions between grammatical knowledge and acquired chunks that language acquisition can be driven forward, otherwise, language acquisition will be inevitably impeded.

\section{Conclusion}

The above research results bear the following three implications for Chinese college English learning of lexical acquisition. First, teachers should emphasize language use in their daily teaching. There are various ways to achieve this, for instance, a substantial increase in language contact and frequency by encouraging language exposure both outside and inside the classroom, stimulation of mutual accessibility of nodes in learners' lexical network, and cultivation of learners' language productive ability. Second, special attention should be paid to the relevance between earlier and later acquired items. Earlier acquired words or collocations should be made full use of in the process of the acquisition of later ones, so that AoA effects can be minimized. Lexical acquisition can be less time-consuming and less word-by-word translation oriented. Finally, it deserves reflection on the fact that lexical acquisition is far from our expectation in college English learning and teaching. It is not necessarily true that pedagogical value, high frequency, early age of onset and the ability to pass authoritative exams lead to ultimate acquisition. Ways of presentation and methods to build up relevance between different acquisition stages still remain to be explored in classroom-based Chinese EFL at the college level.

As an explorative study, this research studies AoA effects in Chinese EFL learners' acquisition of delexicalized verbs and collocations, aiming to provide evidence and support for the learning and teaching of highly frequent words and collocations in the long process of classroom-based compulsory language acquisition. However, it must be pointed out that the present study still has limitations. In our study, special attention is paid to pedagogical values and different stages of acquisition, but at the expense of certain neglect of age of onset and the interactional influence of frequency on lexical acquisition. Further research may be experimental and explore the relationship of age and frequency in lexical acquisition. Subjects in the present study are Chinese EFL learners who began their classroom-based English learners from primary school. Thus studies on subjects of other types or proficiency levels are called for, because they can enable more in-depth understandings of AoA effects. As for how AoA effects can be avoided, it is still in controversy, and requires further investigation.

\section{References}

Chen, B. G., You, W. P. \& Wang, L. X. (2006). The research development of the age-of-acquisition effect in lexical processing. Psychological Science, 29 (6): 1515-1517.

Gui, S. C. (2007). Uncertainty judgment and the acquisition of delexicalized verbs by Chinese learners of English. Foreign Language Teaching and Research, 39(1):3-11.

Lu, Z., \& Tu, L. (2010). The L2 acquisition age and the proficient Chinese-English bilinguals' mental lexicon representation: evidence from a bilingual stroop task . Journal of Foreign Languages, 33(4):47-54.

Wang, C.M. (2013) Age in second language acquisition. Retrieved on October, 20, 2013 from http:// wenku.baidu.com/view/7234dc10a216147917112809.html.

Xiao, Y. N. \& Dai, M. C. (2004). Issues in application of SLA findings to classroom instruction. Foreign Language World, 101 (3): 32-39.

Zhao, F. \& Zou, W. C. (2008). A narrative study of the age of onset and its implications for foreign language teaching. Modern Foreign Language, 31 (3): 317-327.

Altenberg, E., \& Cairns, H. (1983). The effects of phonotactic constraints on lexical processing in bilingual and monolingual subjects. Journal of Verbal Learning and Verbal Behavior, 22 (2): 174-188. DOI: 10.1016/S00225371(83)90134-2

Birdsong, D. (2006). Age and second language acquisition and processing: a selective overview. Language Learning, 56 (s1): 9-49. DOI: 10.1111/j.1467-9922.2006.00353.x

Christiansen, M. H., \& Charter, N. (2001). Connectionist Psycholinguistics in Perspective. In M. H. Christiansen \& N. Charter, Connectionist Pyscholinguistics. Westport, CT: Ablex, 19-75.

Coltheart, V., Laxon, V. J. \& Keating, C. (1988). Effects of word imageability and age of acquisition on children's reading. British Journal of Psychology, 79 (1): 1-12. DOI: 10.1111/j.2044-8295.1988.tb02270.x

Collins Cobuild English Grammar. (1990). Collins: London and Glasgow.

Ellis, A., \& Morrison, C. (1998). Real age-of-acquisition effects in lexical retrieval. Journal of Experimental Psychology: Learning, Memory, and Cognition, 24 (2): 515-523. DOI: 10.1037/0278-7393.24.2.515

Gilhooly, K. J. \& Gilhooly, M. L. (1980). The validity of age-of-acquisition ratings. British Journal of Psychology, 71 (1): 105-110. DOI: 10.1111/j.2044-8295.1980.tb02736.x

Lewis, M. (1997). Implementing the Lexical Approach: Putting Theory into Practice. Hove: Language Teaching Publications. 
Liu, Y. et al. (2008). Age-of-acquisition effects on oral reading in Chinese. Psychonomic Bulletin \& Review, 15 (2): 344-350. DOI:10.3758/PBR.15.2.344

Myles, F., Mitchell, R. \& Hooper, J. (1999). Interrogative chunks in French L2: a basis for creative construction. SSLA, 1999, 21(1):49-80. DOI: http://dx.doi.org/

Sinclair, J. (1991). Corpus, Concordance, Collocation . Oxford: OUP.

Webb, S. (2008). Receptive and productive vocabulary sizes of L2 learners. SSLA, 30 (1): 79-95. DOI: http://dx.doi.org/10.1017/S0272263108080042

Zevin, J. D., \& Seidenberg, M. S. (2002). Age of acquisition effects in word reading and other tasks. Journal of Memory and Language, 47 (1): 1-29. doi:10.1006/jmla.2001.2834 